\title{
Childhood bacterial meningitis: antimicrobial use pattern and treatment outcomes: a prospective observational study
}

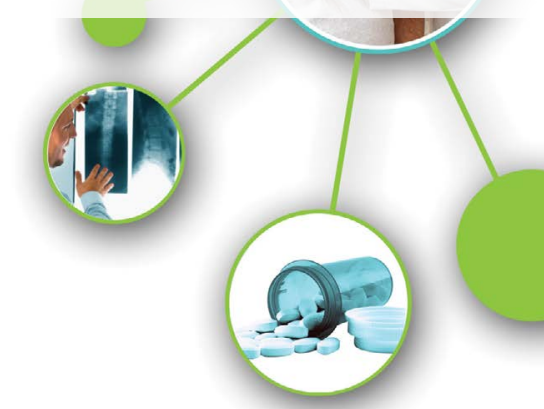

Background: Bacterial meningitis continues to be an important source of mortality and morbidity in infants and children throughout the world despite advances in antibiotics. Resource limited countries contribute more to the mortality and morbidity. We conducted this study to assess antimicrobial use pattern and determine treatment outcomes among children hospitalized with bacterial meningitis.

Study design: Prospective observational study was conducted among infants and children admitted to pediatric ward of Jimma University Specialized Hospital. The data was collected with pretested questionnaire and entered into Epi Data (version 3.1), then exported to SPSS (version 20.0) for analysis. Logistic regression analysis was made to determine independent predictors of poor outcomes.

Results: A total of 89 samples of patients which were treated for bacterial meningitis were analyzed. Ampicillin plus Gentamycin was used as initially antibiotic regimen in most young infants (86.8\%); while majority of older infants and children (66.7\%) initially managed with crystalline penicillin plus Chloramphenicol. Among patients those were treated, $67.4 \%$ improved without acute complication, while the remaining $32.6 \%$ had poor outcomes $(9 \%$ died, $18 \%$ had delayed fever and $5.6 \%$ had acute neurologic complications). Change of antibiotics from empiric therapy was found to be independent predictor of poor outcomes in young infants ( $\mathrm{AOR}=4.42,95 \% \mathrm{Cl}(1.01-19.44)$ ). However, in older infants and children: irritability (AOR=38.39, 95\% Cl (1.78-829.36)) and seizure prior to admission (AOR=27.53, 95\% Cl (1.45-522.35)), initial antibiotic regimen with ceftriaxone plus gentamycin ( $A O R=66.48,95 \% \mathrm{Cl}(3.16-1400.13)$ ), and missed doses of antibiotics ( $A O R=47.33,95 \% \mathrm{Cl}(2.14-1046.19)$ ) were found to independently predict poor outcomes.

Conclusion: The antimicrobials use pattern in this study was nearly similar with the recommendation of national guideline. At discharge nearly one-fourth of the patients treated for bacterial meningitis experienced poor outcomes implying need revising management protocol of childhood bacterial meningitis.

\section{Keywords: childhood, bacterial meningitis, antimicrobials, poor outcomes, Ethiopia}

\section{Introduction}

Meningitis is one of the most common types of central nervous system (CNS) infection. It is an inflammation of the meninges; that involves the subarachnoid space or spinal fluid [1]. Even though there are a number of causes of meningitis (like bacterial, viral and fungal), bacterial meningitis is one of the most potentially serious infections occurring in infants and children [2]. Bacterial meningitis (BM) is a severe, potentially life threatening infection that is associated with high rates of mortality, morbidity and significant disability in survivors [3]. In most resource limited settings the mortality of untreated BM approaches 100\% [4-8], and despite the availability of newer antibiotics and preventive strategies, these morbidity and mortality due to BM continued the same in the past two decades [3,9-12].

Globally, BM affects approximately 1.2 million people each year, with more than two thirds of these occurring under 5 years of age [7]. It causes almost 170,000 deaths per year [8] and as many as $50 \%$ of survivors experience neurological sequelae [9]. In recent years mortality rates related to BM have been increasing and estimated to range from $20 \%$ to $25 \%$ [8]. WHO reported BM as an important cause of childhood morbidity and mortality apart from the five major killer diseases of children under five years (acute respiratory infections mostly pneumonia, diarrhea, measles, malaria and malnutrition) [10].
Habtamu Acho Addo ${ }^{1 *}$, Sadikalmahdi Hussen ${ }^{2} \&$ Legese chelkeba ${ }^{3}$

${ }^{1}$ Department of Pharmacy, College Health Sciences, Mizan-Tepi University, Mizan-Teferi, Ethiopia

${ }^{2}$ School of Pharmacy, College Health Sciences, Addis Ababa University, Addis Ababa, Ethiopia

${ }^{3}$ School of Pharmacy, Institute of Health Sciences, Jimma University, Jimma, Ethiopia

*Author for correspondence:

achuhab31@gmail.com 
In general, the occurrence of adverse consequences of $\mathrm{BM}$ in developed countries is strongly reduced by vaccination strategies, advances in antibiotic treatment, and good care facilities. In contrast, those resource limited countries are exposed to a number of factors that lead them to be vulnerable to those consequences of BM. To mention some: (a) Non-implementation of vaccination programs against major meningeal pathogens; (b) Late presentation of patients, having been given antibiotics without a definite diagnosis in primary or private settings, consequently, many CSF samples do not show the causative agent; (c) Late and insufficient CSF culture and Gramstaining results even though they are basic for definitive diagnosis and guiding treatment; (d) Many hospitals cannot afford expensive third generation cephalosporin and rely on chloramphenicol and penicillin as the first-line antibiotic treatment for meningitis; and (e) Intensive care units are few and not always well staffed [11-15].

In African, children experience both the highest incidence rates [12] as well as the consequences of BM in the world [13].

In Ethiopia, BM alone accounts for about 6-8\% of all causes of the hospital admissions and the case fatality rates associated with it is as high as $22-28 \%$. It has remained a serious health concern in Ethiopia too for the past few decades.

The consequences of all these lead to a considerable emotional, financial and human resource burden on the family as well as the health care system [15-18].

The causes of BM vary with ages in pediatrics. The most common bacterial causes of neonatal meningitis are Listeria monocytogenes (LM), Group B streptococcus (GBS) and Escherichia coli (E. coli) [19-22]; that are mainly acquired from the maternal birth canal during delivery. Whereas, the commonest etiologic agents in children beyond the neonatal period are: Haemophilus Influenzae Type b (HIb), Neisseria meningitidis and Streptococcus pneumoniae [16]. However, these are not the only organisms limited to pediatrics; alterations of host defense due to anatomic defects or immune deficits also increase the risk of meningitis from less common pathogens such as Pseudomonas aeruginosa and Staphylococcus aureus [23-26].
Appropriate empiric antimicrobial treatment should be initiated as soon as possible after the diagnosis is considered to reduce the risk of mortality and complications due to delay in treatment [27]. The choice of empiric antibiotics should take into consideration blood brain barrier [BBB] penetration, the local epidemiology, early versus late disease, resistance patterns and availability within resource constraints [28].

In resource limited settings the treatment of pediatric BM generally has two protocols based on age (under 2 months and above 2 months of age) [29]. Accordingly, for neonates and young infants (under 2 months of age) the first-line antibiotics are Ampicillin and Gentamicin and alternatives, a third-generation cephalosporin, such as Ceftriaxone [20] or Cefotaxime plus Gentamycin [30]. For infants and children (above 2 months of age) the first line is the combination of Penicillin G and Chloramphenicol and the alternative is Ceftriaxone [31-33], or Cefotaxime [16]. For patients not responding to the first line regimens, vancomycin plus ceftazidime can be considered.

Although the major burden of childhood BM occurring in the developing world, the most currently existing literature originated from wealthy countries [34]. As far as we know, limited studies have been done in our country regarding prevalence, etiology, diagnosis, antimicrobial sensitivity, and outcomes of meningitis [24-30]; however studies concerning treatment and its outcomes are still lacking. Therefore, the need for further study in our set up was unquestionable to assess antimicrobial use pattern and determine predictors of poor outcomes of childhood BM.

\section{Methods}

\section{- Study design, period and setting}

From February 25 to April 29 2015, a prospective observational study was conducted at the pediatric ward of Jimma University Specialized Hospital (JUSH). JUSH is found in Jimma Town which is located $335 \mathrm{Km}$ Southwest of Addis Ababa, the capital of Ethiopia. Currently JUSH is the only teaching and referral hospital in the southwestern part of the country. It has 450 beds and more than 750 staff of both professional and supportive. It provides services in different specialty areas for more than 15 million people of the catchment population [35]. 


\section{Case identification \\ - Inclusion criteria}

All pediatrics cases (01 day to 14 years of age) that were clinically suspected or confirmed as meningitis and started treatment for $\mathrm{BM}$ during the study period were included in the study. Meningitis was suspected if the patient was presented with any of the following signs of serious bacterial infection: lethargic, vomiting ( $\geq 3$ episodes), decreased feeding or inability to breast feed, bulging fontanel ( $\leq 2$ years), irritable, high-pitched cry, fever (auxiliary measurement $\geq$ $38^{\circ} \mathrm{C}$ ), and headache (above 2 year), meningeal irritation signs (Kernig or Brudzinski signs or neck stiffness, $\geq 1$ year). The presence of seizure, impaired consciousness (Blantyre Coma Scale $<4$ if $<9$ months of age and $<5$ if $\geq 9$ months of age), signs of raised intracranial pressure, unequal pupils, focal paralysis in any of the limbs, and irregular breathing on examination were considered critical for suspicion of meningitis [36]. However, lumbar puncture was performed to confirm the diagnosis after initiating antibiotic treatment once the infant has been stabilized.

\section{- Exclusion criteria}

- Patients lost to follow within 7 days after starting treatment, and

- Children, in whom the initial diagnosis changed to others than BM like fungal, viral after they were included in the study.

\section{Ethical considerations}

Official ethical clearance was obtained from the institutional review board of JU, college of health sciences, and permission from the medical director of the hospital prior to data collection. A written patient consent was obtained from care givers or family in 2 local languages (Afan Oromo and Amharic) prior data collection. From the very beginning, they were assured that no personal identity would be disclosed, their participation was completely voluntary and that they could be free to withdraw at any time, and this could not affect the medical care that would be given to their child. Above all, the study procedures did not cause any harm to the patient.

\section{Study definitions}

Young infants: were defined in this study as those infants under 2 months of age; and older infants and children: were those infants and children whose age ranged from 2 months to 14 years based on the treatment protocol $[5,8]$. Therefore, Pediatrics according to the current study, included infants and children aged from 1 day to 14 years.

Bacterial meningitis were defined according to physician's clinical diagnosis, including either laboratory-confirmed or probable cases and if no changes in treatment considered until discharge or death to other causes of meningitis like fungal, tuberculosis.

Short term treatment outcomes: according to this study was defined as outcomes of BM detected only until discharge. These included: good and poor outcomes.

- Good outcome- which means improvement without acute complications

- A sign of improvement: normalization of fever was considered as an indicator of improvement from BM since fever is the single most common presenting complaint in patients with BM [37]. In this study delayed fever was defined as fever persisted for more than 7 days [38]. For afebrile patients, other clinical features they presented with were followed for improvement.

- Poor outcome- death within the ward, delayed fever, and developed acute neurologic complications during treatment or at discharge.

- Acute complication: was defined as any complication of BM detected until discharge.

Data collection and quality assurance

First, the data collectors (two hospital pharmacists) and a supervisor (pharmacist) were trained for two days before data collection. Since the focal point of the study was to insure the actual picture of the setup, all the data collectors, the executive program and the principal investigator restricted from interfering the management in the ward. They stuck with each patient twice a day (sunrise and afternoon) recording pertinent data based on the variables on the questionnaires. The supervisor and principal investigator thoroughly followed and coordinated the overall activities.

Pretest was performed and the instruments were modified accordingly, but the pretested samples were not included in the analysis. Frequencies were used to suss out for entry 
errors, missed values and outliers. Any error identified is rectified immediately by revising the original data using the unique code.

\section{Statistical analysis}

The gathered data were entered into Epi data (version 3.1) and exported to statistical package for social science (SPSS) Version 20 for analysis. Discrete variables are expressed as counts (percentage) and continuous variables as means \pm standard deviation (SD) or median and interquartile range (IQR). All continuous data were categorized based on standard cut off points, or mean / median to fit for logistic regression.

Univariate analysis was done for all independent variables to select possible candidates for multivariate logistic regression and the criterion for selection was $\mathrm{p}<0.25$ from the univariate analysis. Lastly, an odds ratio (OR) and $95 \%$ confidence interval were employed to determine the precision of the study and the level of statistical significance was considered at p- value $<0.05$.

For the sake of analysis the main treatment outcomes of BM were categorized as good and poor outcomes. Risk factors of poor results for young infants and that of older infants and children might be different since they were disclosed to different treatment regimens. Hence, a separate analysis was done in the two groups and introduced as follows.

I) Risk factors for poor outcomes of BM in young infants

In order to determine their association with the incidence of poor outcomes, 5 potentially relevant predictors were chosen for multivariate analysis based on their significance from the univariate analysis with $\mathrm{p}<0.25$ (TABLE 5). These were: (1) male gender, (2) mode of delivery, (3) severe dyspnea, (4) any antibiotic changes and (5) number of $\mathrm{AB}$ doses missed. In that location were no missing data on the 5 variables selected. Among the five possible risk factors, multivariate logistic regression identified only one, any antibiotic change to be an independent predictor of the poor outcomes.

II) Risk factors for poor outcomes of BM in older infants and children

The following seven nominees were chosen based on their p-value from univariate analysis results (TABLE 6). Factors with $\mathrm{p}<0.10$ were selected, because the sample size for this age group was small $(\mathrm{n}=36)$ to allow higher $\mathrm{p}$-value $(<0.25)$ that would include more candidates. In that location were no missing data for all the seven selected variables. These were: (1) male gender, (2) irritability and (3) seizure prior to admission, (4) presence of any comorbidity, (5) initial $A B$ regimen with Ceftriaxone plus Gentamycin or Ceftriaxone alone instead of Crystalline penicillin plus Chloramphenicol, (6) longer than 1 hour delay to treatment initiation from diagnosis and (7) missing one or more doses of $\mathrm{AB}$ during the handling course. From the seven potential risk factors, multivariate logistic regressions determined the following four independent predictors of poor outcomes of BM: (1) irritability and (2) seizure prior admission, (3) initial $A B$ regimen with Ceftriaxone plus Gentamycin instead of Crystalline penicillin plus Chloramphenicol, and (4) missing 1or 2 doses of $\mathrm{AB}$ during the treatment course compared to patients never missed.

Furthermore, multi-collinearity diagnostic test was done for all predictors of poor outcomes of childhood BM to see their impact in the regression model and the variance inflation factors (VIF) was utilized to assess the impact of collinearity among the variables. HosmerLemeshow test was employed to examine the goodness-of-fit of the logistic regression model.

\section{Results}

Demographic and baseline characteristics of the patient

A total of 693 patients (285 young infants and 408 older infants and children) were admitted to the pediatric ward of JUSH during the study period. Among these, 102 patients diagnosed as meningitis and started treatment for BM. Thirteen patients were excluded from follow up due to the following reasons; 4 lost to follow up before 7 days of treatment, in 2 patients physician decided to stop therapy and in 7 patients diagnosis changed. Therefore, the analysis was done for a total of 89 patients that completed the whole course of treatment for $\mathrm{BM}$ and die within the ward after initiation of treatments for meningitis (FIGURE 1).

The basic demographic and baseline characteristics are summarized in TABLE 1 . The median age of young infants was 6 days; whereas 
older infants and children it was 27 month. The proportion of males were higher in both age groups; 66\% (young infants) and 61.1\% (older infants and children).

The median durations of illness before

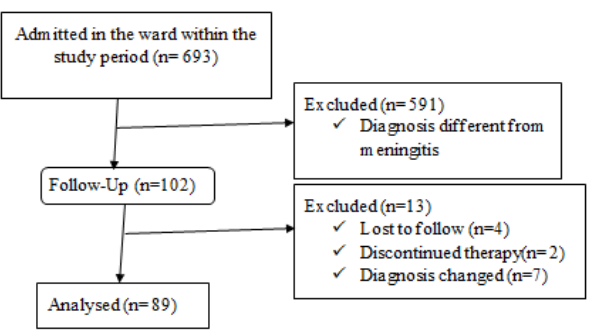

FIGURE 1. Flow Diagram showing enrollment of children treated for BM in JUSH during February 25- April 29, 2015. hospital presentation were 24 hours with IQR (7.0-72.0) for young infants, whereas, 72 hours with IQR (24.0-96.0) for older infants and children (TABLE 1). The two most common clinical characteristics at presentation for young infants were: inability to breast feed $(84.9 \%)$ and fever $(69.8 \%)$, followed by vomiting $(49.1 \%)$ (FIGURE 2).

Nevertheless, the most usual features for older babies and children were fever (94.4\%) and vomiting $(80.6 \%)$, followed by headache, decreased feeding and seizure (each 61.1\%) (FIGURE 3).

Lumbar puncture was done in 60 patients $(67.4 \%)$ and conked out in the remaining 29 (32.6\%) of cases. Among these, the causative

\section{Table 1. Age distribution of measles from 2010 to 2016.}

\begin{tabular}{|c|c|c|c|}
\hline Patient characteristics & $\begin{array}{c}\leq 2 \text { months } \\
(n=53), N(\%)\end{array}$ & $\begin{array}{c}>2 \text { months } \\
(n=36), \mathrm{N}(\%)\end{array}$ & $\begin{array}{c}\text { Total }(n=89) \\
N(\%)\end{array}$ \\
\hline Age, median months (IQR) & $0.2(0.03-0.93)$ & $27(9.8-72.0)$ & \\
\hline \multicolumn{4}{|l|}{ Gender } \\
\hline Male & $35(66)$ & $22(61.1)$ & $57(64.0)$ \\
\hline Female & $18(34)$ & 14(38.9) & $32(36.0)$ \\
\hline Birth weight $(\mathrm{kg})$, mean $( \pm \mathrm{SD})$ & $2.8(2.2-3.4)$ & & \\
\hline$\geq 2.5$ & $25(75.8)$ & & \\
\hline $1.5-2.5$ & $6(18.2)$ & & \\
\hline$\leq 1.5$ & $2(6.0)$ & & \\
\hline \multicolumn{4}{|l|}{ Gestational age(in weeks) } \\
\hline$>37$ & $37(69.8)$ & & \\
\hline$\leq 37$ & $12(22.6)$ & & \\
\hline \multicolumn{4}{|l|}{ Mode of delivery } \\
\hline Vaginal & $44(83)$ & & \\
\hline Caesarian Section & $9(17)$ & & \\
\hline \multicolumn{4}{|l|}{ Immunization history } \\
\hline Fully immunized & & $19(52.8)$ & \\
\hline Not fully immunized & & $17(47.2)$ & \\
\hline \multicolumn{4}{|l|}{ Previous history of hospital admission } \\
\hline Yes & 0 & $3(8.3)$ & $3(3.3)$ \\
\hline No & $53(100)$ & $33(91.7)$ & $86(96.7)$ \\
\hline \multicolumn{4}{|l|}{ Maternal history of fever or UTI } \\
\hline Yes & $12(22.6)$ & & \\
\hline No & $41(77.4)$ & & \\
\hline Median duration of symptoms (hrs.), (IQR) & $24(7.0-72.0)$ & $72(24-96)$ & \\
\hline Mean body temperature $\left({ }^{\circ} \mathrm{C}\right)$ at admission $( \pm \mathrm{SD})$ & $37.6(36.7-38.5)$ & $38.3(37.4-39.2)$ & \\
\hline Lumbar puncture was done & $29(54.7)$ & $31(86.1)$ & $60(67.4)$ \\
\hline Pneumococci & $1(1.9)$ & $2(5.6)$ & $3(3.4)$ \\
\hline Meningococci & & $1(2.8)$ & $1(1.1)$ \\
\hline Hlb & & $3(8.3)$ & $3(3.4)$ \\
\hline No microorganisms seen & $20(37.7)$ & $16(44.4)$ & $36(40.4)$ \\
\hline
\end{tabular}




\begin{tabular}{|c|c|c|c|}
\hline \multicolumn{1}{|c|}{ Lumbar puncture failed } & $24(45.3)$ & $5(13.9)$ & $29(32.6)$ \\
\hline No reagent & $6(11.3)$ & $11(30.6)$ & $17(19.1)$ \\
\hline CSF WBC count was done & $26(49.0)$ & $27(75.0)$ & $53(59.6)$ \\
\hline CSF WBC count/mm ${ }^{3}$, median(IQR) & $2(0.0-11.2)$ & $2(0.0-12.0)$ & $2(0.0-11.0)$ \\
\hline CSF protein analysis was done & $10 / 53$ & $10 / 36$ & $20(22.5)$ \\
\hline CSF protein (mg/dl), median (IQR) & $64.0(32.0-128.0)$ & $96.0(28.0-158.0)$ & $\begin{array}{c}80.0(32.0- \\
150.0)\end{array}$ \\
\hline CSF glucose analysis was done & $4 / 53$ & $5 / 36$ & $9 / 89(10.1)$ \\
\hline CSF glucose (mg/dl), median(IQR) & $30.9(14.8-39.8)$ & $32.5(18.9-39.3)$ & $32.5(18.9-39.3)$ \\
\hline Presence of comorbidities & & & \\
\hline \multicolumn{1}{|c|}{ No } & $37(69.8)$ & $21(58.3)$ & $58(65.2)$ \\
\hline Sepsis & $16(30.2)$ & $15(41.7)$ & $31(34.8)$ \\
\hline Malaria & & & \\
\hline Types of comorbidities & $34(91.9)$ & $6(28.6)$ & $40(44.9)$ \\
\hline and anemia) & & $5(23.8)$ & $5(5.6)$ \\
\hline Others (pertussis, HIV, impetigo, moderate diarrhea, SAM & $3(8.1)$ & $10(47.6)$ & $13(14.6)$ \\
\hline
\end{tabular}

SD- standard deviation, IQR- interquartile range, UTI- urinary tract infection, HIV-human immunodeficiency virus, SAM-sever acute malnutrition, CSF- cerebrospinal fluid

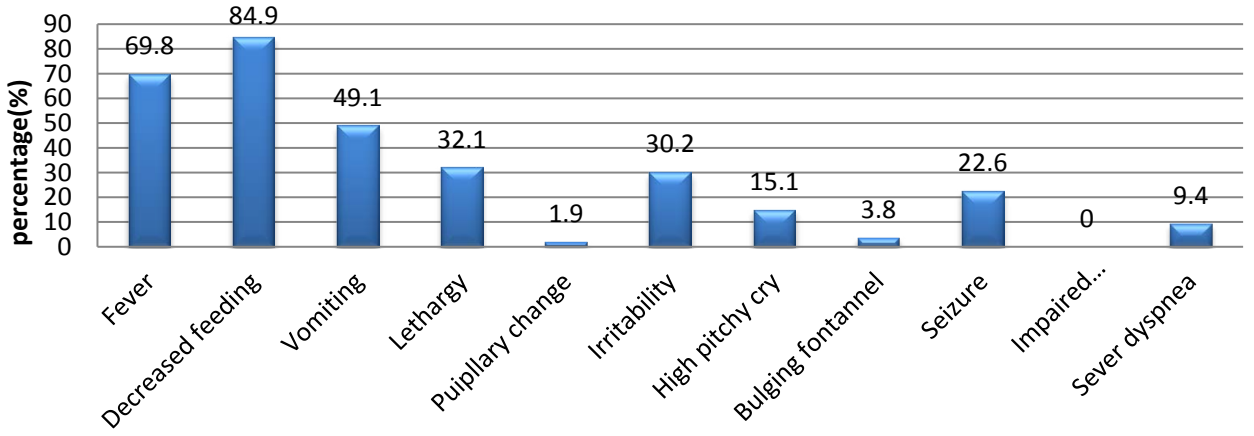

types of clinical presentations

FIGURE 2. Clinical presentations prior to admission of younger infants treated for BM in JUSH during February 25- April 29, 2015.

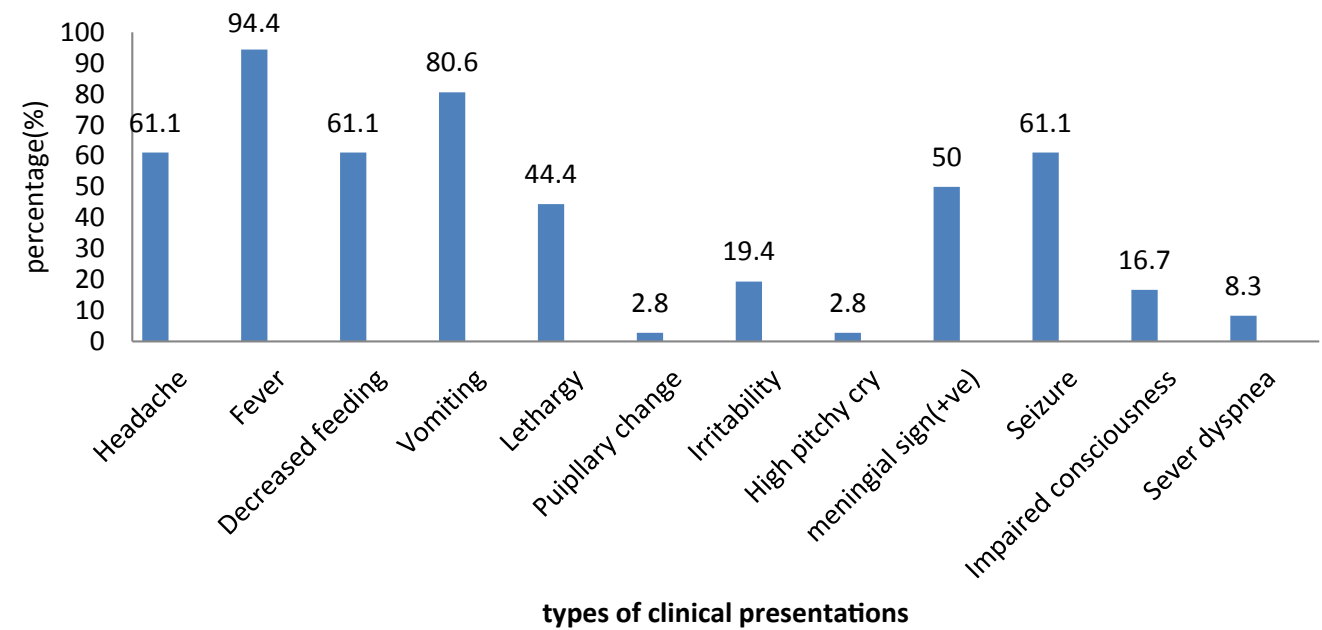

FIGURE 3. Clinical presentations prior to admission of older infants and children treated for BM in JUSH during February 25- April 29, 2015.

pathogens were identified by CSF stain only in 1 meningococcal. In 36 patients (40.4\%), the 7 patients (7.9\%): 3 pneumococcal, $3 \mathrm{HIb}$ and result was reported as 'no microorganism was 
seen', and in the remaining 17 patients (19.1\%) there were no reagents to carry out the stain (TABLE 1).

From all pediatric patients admitted in this ward during the study period, $14.4 \%$ were diagnosed with bacterial meningitis (FIGURE 1). Most of the young infants $(69.8 \%)$ had comorbidities, mainly of sepsis (34/37); whereas in older infants and children comorbidities of different types observed in $58 \%$ of the cases (TABLE 1).

\section{Drug related factors}

Factors related to drugs used for the management of childhood BM in the study population are summarized in TABLE 2 . Most of the young infants $(86.8 \%)$ were initially treated with empiric Ampicillin plus Gentamycin regimen. On the other hand, in the majority of the older infants and children (66.7\%), the initial $A B$ regimen was crystalline penicillin plus Chloramphenicol. The median duration of treatment from diagnosis for both age groups was similar, 1 hour with IQR (0.33.0) for young infants and (0.5-2.0) for older infants and children. By considering the delayed presentation and delay in hospital for treatment, these two durations were added to give another important factor, delay of treatment for initial illness. Therefore, the median delay for treatment from initial illness was 32 hours (10.2-73.5) for young infants, whereas 73 hours (25.4-98.4) for older infants and children.

\section{Treatment outcomes of childhood BM}

The median duration of improvement after treatment were 6 days (3.0-9.2) for young infants, whereas 4 days (3.0-7.0) for older infants and children. Nearly $72 \%$ of the young infants and $61 \%$ of older infants and children improved within 7 days of treatment. Altogether, $67.4 \%$ of the patients improved without acute complication; while, $9 \%$ died, $18 \%$ had delayed fever and 5.6\% had acute neurologic complications during their entire stay in the hospital. The observed acute neurologic complications were hemiparesis and recurrent seizures in 3 patients, hearing impairment in 2 patients, and quadriparesis, craniocytosis, and vision impairment each in a patient (TABLE 3).

In general, a total of 69 patients (77.5\%) improved, 8 (9\%) died, $5(5.6 \%)$ referred for further management of the neurologic complications, and $7(7.9 \%)$ withdrew from the treatment with a delayed fever (poor condition) for unknown reasons. Out of a total of 16 patients with delayed fever (TABLE 3), 9 patients later improved due to further

Table 2. Drug regimen used in children treated for BM in JUSH during February 25- April 29, 2015.

\begin{tabular}{|c|c|c|c|c|}
\hline \multicolumn{3}{|l|}{ Regimens } & $\begin{array}{l}\text { Under } 2 \text { months } \\
(n=53), N(\%)\end{array}$ & $\begin{array}{l}\text { Above } 2 \text { months } \\
\qquad(n=36), N(\%)\end{array}$ \\
\hline \multirow{4}{*}{ Initial regimen } & \multicolumn{2}{|l|}{ Ampicillin plus Gentamycin } & $46(86.8)$ & $1(2.8)$ \\
\hline & \multicolumn{2}{|l|}{ Ceftriaxone plus Gentamycin } & $7(13.2)$ & $9(25.0)$ \\
\hline & \multicolumn{2}{|c|}{ Crystalline Penicillin plus Chloramphenicol } & - & $24(66.7)$ \\
\hline & \multicolumn{2}{|l|}{ Ceftriaxone alone } & - & $2(5.6)$ \\
\hline \multirow{3}{*}{$\begin{array}{l}\mathrm{R} \text { e } \mathrm{g} \text { i } \mathrm{m} \text { e } \mathrm{n} \\
\text { changed to }\end{array}$} & \multicolumn{2}{|l|}{ Ceftriaxone plus Gentamycin } & $13(24.5)$ & - \\
\hline & \multicolumn{2}{|l|}{ Ceftriaxone alone } & - & $2(5.6)$ \\
\hline & \multicolumn{2}{|l|}{ Ceftazidime plus Vancomycin } & $2(3.8)$ & $1(2.8)$ \\
\hline \multicolumn{3}{|c|}{ Median duration (hours) to treatment from diagnosis(IQR) } & $1(0.3-3.0)$ & $1(0.5-2.0)$ \\
\hline \multicolumn{3}{|c|}{ Mean duration to $\mathrm{AB}$ change (days, $\pm \mathrm{SD}$ ) } & $5(2.0-8.0)$ & $3.2(1.4-5.0)$ \\
\hline \multicolumn{3}{|c|}{ Median delay of treatment from initial illness(IQR) } & $32(10.2-73.5)$ & $73(25.4-98.4)$ \\
\hline \multirow{2}{*}{\multicolumn{2}{|c|}{ Number of $A B$ doses missed }} & 1 or 2 & $8(66.7)$ & $9(25.0)$ \\
\hline & & $\geq 3$ & $4(33.3)$ & $2(5.6)$ \\
\hline \multirow{2}{*}{\multicolumn{2}{|c|}{ Reasons for missing }} & Unaffordability & $9(75.0)$ & $11(100)$ \\
\hline & & IV line unavailable & $3(25.0)$ & 0 \\
\hline \multicolumn{3}{|c|}{ Dexamethasone use } & - & $16(44.4)$ \\
\hline \multirow{2}{*}{\multicolumn{2}{|c|}{$\begin{array}{l}\text { Duration to dexamethasone with reference to } \\
\text { Abs }\end{array}$}} & $\begin{array}{l}\text { At same time or within } \\
20 \text { minutes before } A B s\end{array}$ & - & $10(62.5)$ \\
\hline & & $\begin{array}{l}\text { Beyond } 20 \text { minutes or } \\
\text { after Abs }\end{array}$ & - & $6(37.5)$ \\
\hline
\end{tabular}


management and supportive care given within the ward (TABLE 4).

\section{Predictors of poor outcomes of BM in young infants}

Fifteen patients out of 53 young infants treated for BM $(28.3 \%)$ had poor outcomes (TABLE 3). Sixty six percent (35/53) of young infants treated for BM were males (TABLE 1). The occurrence of poor outcomes in young infants was less likely for males compared to females, but it was not statistically significant. (AOR $=0.285,95 \%$ CI (0.068-1.193)). Delivery assisted by caesarean section was done in $31 \%$ infants under 2 months of age (TABLE 1). Infants whose delivery aided by Caesarian section experienced a 2 fold risk of poor outcomes than those delivered vaginally, however the difference was not statistically significant, $A O R=2.015$, 95\% CI (0.292-13.907).

Only 5 patients $(9.4 \%)$ presented with severe dyspnea among young infants (TABLE 1). Patients with severe dyspnea prior admission were 5 times more likely to have poor outcomes than those without severe dyspnea $(A O R=$ 5.335, 95\% CI (0.567-50.209)), however no significant difference observed between the two.
In young infants the frequently used initial regimen has been Ampicillin plus Gentamycin (86.8\%), while only $13.2 \%$ of patients were given Ceftriaxone plus Gentamycin for initial management (TABLE 2). Initial $A B$ regimens were either changed or modified only in 15 patients $(28.3 \%)$ of this age group. Most of the $\mathrm{AB}$ changes were modifications of the initial Ampicillin plus Gentamycin to Ceftriaxone plus Gentamycin $(13 / 15,86.7 \%)$, whereas in only 2 cases the initial $\mathrm{AB}$ regimen was changed to Vancomycin plus Ceftazidime (13.3\%). The risks of experiencing poor outcomes were highly associated with changing the initial $A B s$ than completing the whole course of treatment with the initial $A B s$ with $A O R=4.425,95 \% \mathrm{CI}$ (1.007-19.440). During the course of treatment, 12 patients $(22.6 \%)$ missed at least one dose of their ABs (TABLE 2). Of these: 8 patients missed 1 or 2 doses and 4 patients missed 3 or more doses. Missing 1 or 2 doses of the prescribed $A B s$ was found to increase the risk of experiencing poor outcomes as compared to patients who never missed their entire doses of $\mathrm{ABs}$, however the difference was not statistically significant with AOR $=3.708$, 95\% CI (0.61522.364). Missing 3 or more doses also increased the occurrence of poor outcomes compared to

\begin{tabular}{|c|c|c|c|c|}
\hline \multirow{2}{*}{\multicolumn{2}{|c|}{ Patients Outcomes }} & $\begin{array}{c}\text { For } \leq 2 \text { months } \\
\quad(n=53)\end{array}$ & $\begin{array}{c}\text { For }>2 \text { months } \\
\quad(n=36)\end{array}$ & General $(n=89)$ \\
\hline & & $\mathbf{N}(\%)$ & $\mathbf{N}(\%)$ & N (\%) \\
\hline \multicolumn{2}{|c|}{ Good outcomes/improved } & $38(71.7)$ & $22(61.1)$ & $60(67.4)$ \\
\hline \multirow{3}{*}{ Poor outcomes } & Death & $3(5.7)$ & $5(13.9)$ & $8(9.0)$ \\
\hline & Delayed fever & 10(18.8) & $6(16.7)$ & $16(18.0)$ \\
\hline & Acute neurologic complication & $2(3.8)^{b}$ & $3(8.3)^{c}$ & $5(5.6)$ \\
\hline \multicolumn{2}{|c|}{ Median time to improvement (days, IQR) } & $6(3.0-9.2)$ & $4(3.0-7.0)$ & $5(3.0-8.5)$ \\
\hline
\end{tabular}

Table 4. General status at discharge of children treated for BM in JUSH during February 25- April 29, 2015.

\begin{tabular}{|c|c|c|c|}
\hline \multirow[t]{2}{*}{ Statuses at discharge } & $\begin{array}{l}\text { Young infants } \\
\quad(n=53)\end{array}$ & $\begin{array}{l}\text { Older infants } \\
\text { \& children } \\
(n=36)\end{array}$ & $\begin{array}{l}\text { General } \\
(n=89)\end{array}$ \\
\hline & $\mathbf{N}(\%)$ & $\mathbf{N}(\%)$ & $\mathbf{N}(\%)$ \\
\hline Improved & $44(83.0)$ & $25(69.4)$ & $69(77.5)$ \\
\hline Died & $3(5.7)$ & $5(13.9)$ & $8(9.0)$ \\
\hline Neurologic complication (referred) & $2(3.8)^{b}$ & $3(8.3)^{c}$ & $5(5.6)$ \\
\hline Lost to follow up with delayed fever & $4(7.5)$ & $3(8.3)$ & $7(7.9)$ \\
\hline Median time to death in days, (IQR) & & & $5.0(3.25-6.00)$ \\
\hline Median hospital stay in days (IQR) & $14(10-16)$ & $12(9-15)$ & $13(10-15)$ \\
\hline
\end{tabular}


Table 5. Univariate and multivariate logistic regression of risk factors for poor outcomes of BM in young infants $(n=53)$ in JUSH during February 25- April 29, 2015.

\begin{tabular}{|c|c|c|c|c|c|c|c|}
\hline \multirow{2}{*}{\multicolumn{2}{|c|}{ Variables }} & \multicolumn{2}{|c|}{ Outcomes } & \multicolumn{2}{|c|}{ Univariate results } & \multicolumn{2}{|l|}{ Multivariate } \\
\hline & & \multirow{2}{*}{$\begin{array}{c}\begin{array}{c}\text { Good } \\
(\mathbf{n}=38)\end{array} \\
27\end{array}$} & \multirow{2}{*}{$\begin{array}{c}\begin{array}{c}\text { Poor } \\
(n=15) \backslash\end{array} \\
8\end{array}$} & \multirow{2}{*}{\begin{tabular}{|c|} 
p-value \\
0.224 \\
\end{tabular}} & \multirow{2}{*}{$\begin{array}{c}\text { COR(95\% CI) } \\
0.466(.136-1.598)\end{array}$} & \multirow{2}{*}{$\begin{array}{c}\text { AOR(95\% Cl) } \\
0.285(.068-1.193)\end{array}$} & \multirow{2}{*}{$\begin{array}{r}\text { p-value } \\
0.086\end{array}$} \\
\hline Sex & Male & & & & & & \\
\hline & Female & 11 & 7 & & & & \\
\hline \multirow{3}{*}{$\begin{array}{l}\text { Mode of } \\
\text { delivery }\end{array}$} & CS & 5 & 6 & 0.038 & $4.400(1.008-17.790)$ & $2.015(.292-13.907)$ & 0.477 \\
\hline & Vaginal & 33 & 9 & & & & \\
\hline & No & 26 & 11 & & & & \\
\hline \multirow{2}{*}{$\begin{array}{l}\text { Sever } \\
\text { dyspnea }\end{array}$} & Yes & 2 & 3 & 0.122 & $4.500(.670-30.230)$ & $5.335(.567-50.209)$ & 0.143 \\
\hline & No & 36 & 12 & & & & \\
\hline \multirow{2}{*}{$\begin{array}{l}\text { AB changes } \\
\text { considered }\end{array}$} & Yes & 8 & 7 & 0.069 & $3.281(.913-11.796)$ & $4.425(1.007-19.440)$ & 0.049 \\
\hline & No & 30 & 8 & & & & \\
\hline \multirow{3}{*}{ Missed ABs } & 1 or 2 doses & 5 & 3 & \multirow{2}{*}{0.036} & \multirow{2}{*}{$2.853(1.071-7.601)$} & $3.708(.615-22.364)$ & 0.153 \\
\hline & $\geq 3$ doses & 1 & 3 & & & $12.088(.967-151.128)$ & 0.053 \\
\hline & Never & 32 & 9 & & & & \\
\hline
\end{tabular}

Table 6. Univariate and multivariate logistic regression for risk factors for poor outcomes of BM for older infants and children $(n=36)$ in JUSH during February 25- April 29, 2015.

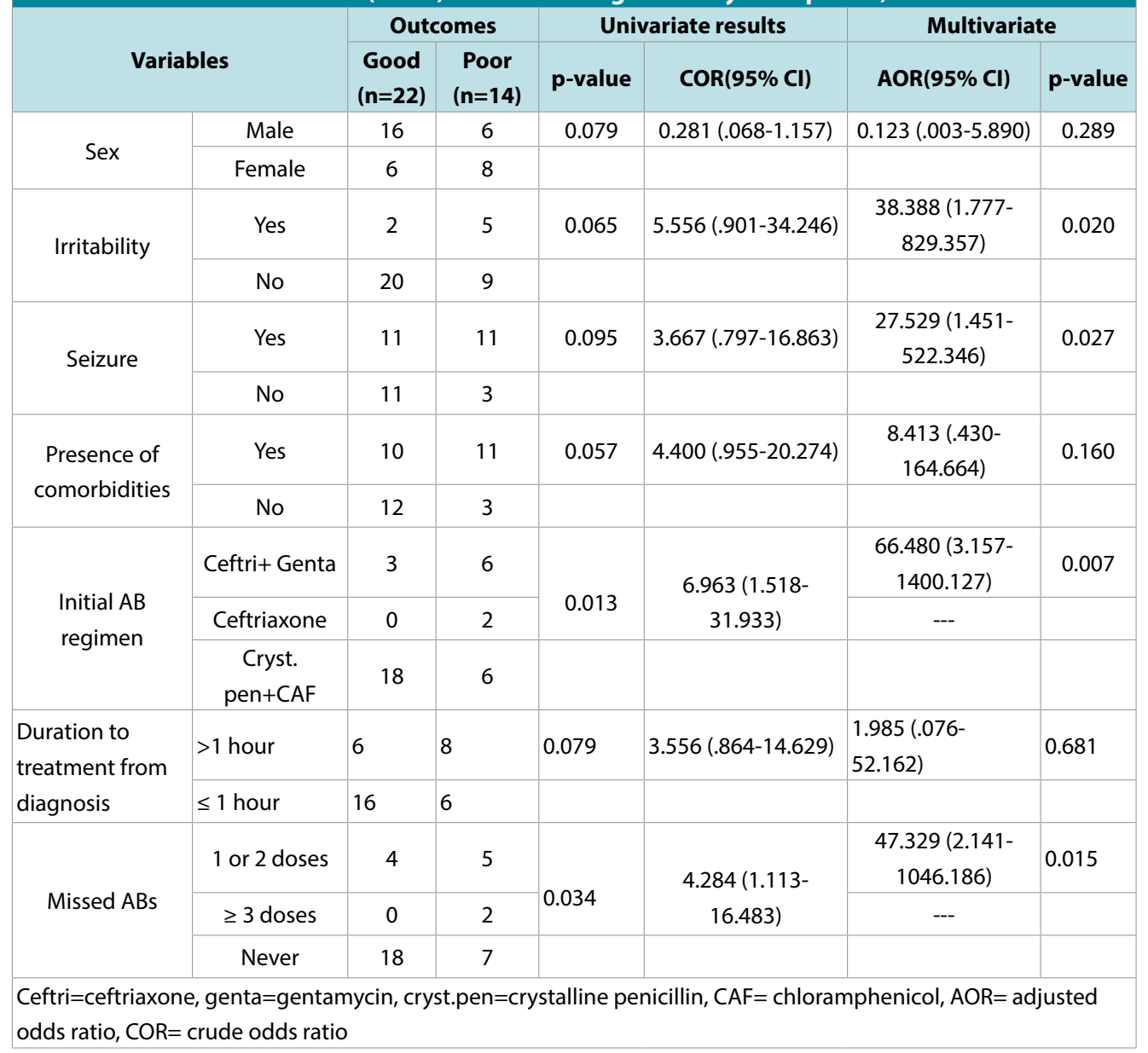

those who never missed their entire doses of $\mathrm{ABs}$, but the statistical significant difference was not observed too, $\mathrm{AOR}=12.088,95 \% \mathrm{CI}$ (0.967-151.128).

\section{Predictors of poor outcomes of BM in older infants and children}

Among the 36 older infants and children treated for BM, 14 patients $(38.9 \%)$ 
experienced poor outcomes (TABLE 3). The majority $(61.1 \%)$ of older infants and children treated for BM were males (TABLE 1). Lower incidence of poor outcomes of BM was observed in males compared to females with $(\mathrm{AOR}=.123$, 95\% CI 0.03-5.890); but the difference was not statistically significant. In older infants and children clinical presentations characterized by irritability was $19.4 \%$ and seizure $61.1 \%$ on admission (TABLE 1). Patients who had an irritable clinical feature prior to admission were at nearly 38.4 times more increased risks of poor outcomes than those without this feature $(\mathrm{AOR}=38$. 388, 95\% CI (1.777-829.357)). Seizure prior admission was also found to be associated with about 27.5 times increased incidence of poor outcomes compared to patients who had not seizure prior admission $(\mathrm{AOR}=27.529,95 \% \mathrm{CI}(1.451-522.346))$.

Twenty one patients among older infants and children (61.1\%) had different types of comorbidities. Of these 6 patients $(28.6 \%)$ had sepsis, 5 patients $(23.8 \%)$ had malaria, and 10 patients $(47.6 \%)$ had either of pertussis, HIV, impetigo, moderate diarrhea, SAM and/or anemia (TABLE 1). Even though the presence of comorbidities was not statistically significant its presence seemed to increase the incidences of poor outcomes $(\mathrm{AOR}=8.413,95 \% \mathrm{CI},(0.430$ 164.664)).

In older infants and children the most commonly used initial $\mathrm{AB}$ regimen was crystalline penicillin plus Chloramphenicol (69.4\%). Ceftriaxone plus Gentamycin and Ceftriaxone alone were also the other initial $\mathrm{AB}$ regimens given to patients of this age group in $25 \%$ and $5.6 \%$ of patients respectively (TABLE 2). An initial regimen with Ceftriaxone plus Gentamycin was found to be a strong predictor of poor outcomes $(\mathrm{p}=0.007)$. As compared to patients who initially treated with Crystalline penicillin plus Chloramphenicol, those initially treated by Ceftriaxone plus Gentamycin were highly associated with increased incidence of poor outcomes $(\mathrm{AOR}=66.480,95 \% \mathrm{CI}$ (3.157-1400.127)). However, longer than 1 hour delay in treatment from diagnosis failed to show statistically significant association with poor outcomes $(\mathrm{AOR}=1.985$, $955 \mathrm{CI}$ (0.07652.162)). During the course of treatment 11 patients $(30.6 \%)$ missed at least one dose of their prescribed ABs. Of these 9 patients missed either 1 or 2 doses, while the remaining
2 patients missed 3 or more doses (TABLE 2). Patients who missed either 1 or 2 doses of $\mathrm{ABs}$ during the entire course of treatment were found to be at about 47 times more risks of experiencing poor outcomes compared to those never missed their doses $(\mathrm{AOR}=47.329,95 \%$ CI (2.141-1046.186)).

\section{Discussion}

Early initiation of an optimal antibiotic therapy for confirmed or suspected BM, pending the CSF results, has been shown to be one of the most important factor to reduce morbidity and lethality $(4,5,9,10,54-57)$. In the current study, most of the young infants $(86.8 \%)$ were initially treated with empiric Ampicillin plus Gentamycin regimen. On the other hand, in older infants and children, the most commonly used empiric $\mathrm{AB}$ regimen was Crystalline penicillin plus Chloramphenicol (66.7\%) followed by Ceftriaxone plus Gentamycin (25\%). The selection and timing of initiation of $\mathrm{ABs}$ were in line with the current recommendation for developing countries [39]. The choice of $\mathrm{ABs}$ was also similar to studies conducted in some resource limited settings. The median duration of treatment from diagnosis for both age groups was the similar, 1 hour. This was similar to the study from Italy (1 hour) and even better than that from Uganda (9.6 hours) [40,41].

Meanwhile, in the course of treatment, in $20 \%$ of patients $\mathrm{ABs}$ changes were considered due to poor response to the empiric regimen. Among young infants for whom $\mathrm{ABs}$ change was considered, in most (87\%) of the cases the empiric Ampicillin plus Gentamycin was modified to Ceftriaxone plus Gentamycin, and only 3 patients the regimen changed to Ceftazidime plus Vancomycin. The change was also in agreement with current recommendations as almost all the changes were made to the first line alternative considering affordability as per the recommendation for resource limited countries of WHO. Guidelines and current evidence recommend narrowing of the empiric regimen as soon as the agent is identified or change of empiric ABs within 2-3 days in cases if it is not possible to identify the agent and the patient is not improving with the empiric $A B s$ [42]. However, the duration to change of $A B s$ in our case was very longer than the recommended. The mean duration of $\mathrm{AB}$ change for young 
infants was 5 days while it was 3.2 days for older infant and children.

Generally, nearly $67 \%$ of patients treated for BM improved within 7 days of treatment without acute complications and the median time for improvement was 5 days. This was better than a study done in Uganda at that time to improve from initiation of treatment was 10.3 days. The difference could be due to delayed presentation (median $=6.5$ days) and late initiation of treatment (median=9.6hours) from Uganda's study compared to median time to hospital presentation was only 2 days and median time to treatment was only 1 hour in the current study. The cumulative incidence of poor outcomes in this study was $32.6 \%$; including mortality of $9 \%$, acute neurologic complications (6\%), and delayed fever in $18 \%$ of patients (TABLE 3). The incidence of poor outcomes was almost comparable with most studies from resource limited settings [43-45] but it could be slightly lower than those reports since only the short-term treatment outcomes were included and had no follow up after discharge that would increase the rate of both mortality and neurologic complications in those studies.

Among patients initially treated with Ampicillin plus Gentamycin, almost $85 \%$ improved without complications and 15\% had poor outcomes while in patients initially treated with a combination of Ceftriaxone and Gentamycin, about $71 \%$ improved without acute complications $29 \%$ experienced poor outcomes. The patients initially treated with Ceftriaxone plus Gentamycin experienced comparatively poorer outcomes, as those patients initially presented with severe clinical feature (like a seizure) at admission.

The incidences of poor outcomes within 7 days of treatment were different when the two age groups compared; nearly $28 \%$ of the young infants versus $39 \%$ of the older infants and children (TABLE 3). The majority of the older infants and children (57\%) presented to the hospital lately (after 2 days of illness), whereas only $36 \%$ of young infants presented after 2 days. However, this difference in delayed presentation with age was not statistically significant on univariate analysis $(\mathrm{COR}=2.3$, $\mathrm{p}=0.061$ ). The median age of the younger infants was 6 days, which might indicate most the young infants were born within the hospital and started treatment immediately as they got ill compared to those older infants and children who came to the hospital after the disease got advanced. Furthermore, the majority of the older infants and children (59\%) had severity characterized by seizure before admission compared to only $23 \%$ of those patients under 2 months (COR=4.9, $\mathrm{p}=0.001)$. Therefore, the higher percentage both by late presentation and severe clinical features before admission could contribute to more incidences of poor outcomes among older infants and children than young infants.

\section{Predictors of poor outcomes in young infants}

In young infants, despite the percentage of males treated was quite higher $(66 \%)$ and those had poor outcome still higher (53\%), the risk of experiencing poor outcomes seemed lower compared to females, but this failed to show statistically significant association in multivariate logistic regression. In the same manner, caesarean section aided delivery initially seemed to increase the risk of poor outcomes compared to vagina delivery, however it was not found to be one of the important predictors of poor outcomes.

In the present study, the presence of severe dyspnea at admission was found to increase the risk of poor outcomes; but statistically significant association with poor outcomes was not determined under multivariate logistic regression. This was similar to the study of Pelkonen et al. in which severe dyspnea before admission showed a non-significant increase in the risks of developing severe neurological sequelae and significantly increased the risk of death [46].

Young infants for whom empiric $\mathrm{AB}$ changed were associated with increased incidence of poor outcomes than those completed their entire course of treatment with the initial $A B$ regimen. First of all, patients for whom $A B s$ changed were those critically ill or those did not improve to the empiric regimen. Secondly, the mean duration of $A B$ change was 5 days, which was much longer than the recommended (2-3 days). Furthermore, most of the patients $(93 \%)$ who $\mathrm{ABs}$ was changed had one or more comorbidities. Therefore, the higher percentage of comorbidities in patients who had $A B$ 
changes could have contributed to the poor outcomes as the difference was significant on univariate logistic regression $(\mathrm{p}=0.026)$, and the longer duration of time to $A B$ changes could also have contributed to. In addition, in some studies, it was highlighted that most of the patients in developing countries have delayed presentation to the higher health care settings having been treated with common 3rd generation cephalosporin (mainly Ceftriaxone) without a definite diagnosis in primary or private settings $[41,47]$. Consequently, these could have its own impact on the sensitivity of the pathogens to these antibiotics, but this needs further investigation. Even though statistically significant difference was not observed, missing one or more doses of the prescribed $A B s$ had increased the risk poor outcomes in the current study. As the main reason for missing was unaffordability, really this needs intervention by improving effective communication of the health care team with patients' family or caregivers and facilitating the opportunities for them on how to get alternative cost effective therapies.

\section{Predictors of poor outcomes in older infants and children}

In this study, despite the gender distribution was predominated by males (61\%), female gender had contributed a higher percentage $(57 \%)$ to the poor outcomes of BM. Interestingly; this gender difference was not statistically significant. Delayed presentation to the hospital (after 72 hours of illness) was higher in females (36\%) versus $27 \%$ in males. Ahmed A. in his study at Gonder and Hawassa highlighted that, there are still social and cultural factors that favor easier access for males to the healthcare facilities as compared to the females and even those females that attend the health care facilities are after the disease got advanced/complicated [3]. This may also highlight the bias or the preference that may be given by the family to the male children over the female children which need further research.

Many clinical features prior to or at hospital admission are associated with the outcome of bacterial meningitis $[47,48]$. In the current study, irritable feature at presentation found to increase the incidence of poor outcomes, as this feature is mostly a manifestation of CNS disorders characterized by abnormal sensitivity signifying the advanced nature of the disease. Similarly, the presence of seizure at or prior to admission was associated with increased incidence of poor outcomes. This is in line with different studies in which the occurrence seizure prior hospital admission increased mortality and sever neurological sequelae, as the occurrence of seizure at presentation indicates the advanced stage (complication) of the disease.

About $58 \%$ of older infants and children treated for BM had one or more comorbidities. These included sepsis in $6(29 \%)$ patients, malaria in $5(24 \%)$, and in 10 children other comorbidities (like pertussis, HIV, impetigo, moderate diarrhea, SAM and/or anemia). The incidence of poor outcomes was $39 \%$ and there were consistently increased incidence of poor outcomes, in both univariate and multivariate logistic regression, in patients who had one or more comorbidities though it was not statistically significant. The possible explanation could be most of these comorbidities were clinical suspicions due to the similarity of clinical features like sepsis and malaria with that of BM rather than laboratory confirmation. Another possible reason that needs further evaluation was; probably there could be proper management of these comorbidities, though the management was not presented here. This was similarly not significant in the study of Prishtina (Kosovo).

The most commonly used initial $\mathrm{AB}$ regimen in older infants and children was crystalline penicillin plus Chloramphenicol (66.7\%) followed by a combination of ceftriaxone and gentamycin (25\%). Out of the 24 patients $(66.7 \%)$ initially treated with crystalline penicillin plus Chloramphenicol, $79.2 \%$ improved without acute complications and the remaining $20.8 \%$ had poor outcomes. However, among 9 patients $(25 \%)$ that were initially given a combination of Ceftriaxone and Gentamycin, almost half (44.4\%) experienced poor outcomes. An initial regimen with Ceftriaxone plus Gentamycin instead of the first line crystalline penicillin plus Chloramphenicol significantly increased the risk of poor outcomes. First of all, the main indication for selection of Ceftriaxone based regimen instead of first line penicillin/ ampicillin based regimen in the ward was severe (critical illness) at presentation. Secondly, in the majority $(75 \%)$ patients initially put on Ceftriaxone plus Gentamycin the initial dose of the drugs was administered lately (after 1 hour of diagnosis) compared to only in $16 \%$ of patients 
on Crystalline penicillin plus Chloramphenicol the drugs were initiated lately (after 1 hour of diagnosis). The reason for the delay could be due to searching of this expensive drug (Ceftriaxone compared to Penicillin) since it is not commonly available in our wards. Thirdly, there were a higher percentage of patients with comorbidities during the course of treatment, $67 \%$ of ceftriaxone based against $53 \%$ for penicillin based. Besides, the use of adjuvant dexamethasone was lower (33\% versus 54\%) and not fully vaccinated were higher $(78 \%$ versus 33\%) in patients on Ceftriaxone based regimen compared to patients on Penicillin based regimen respectively. Therefore, the increased incidence of poor outcomes among patients on a Ceftriaxone based regimen than those on Penicillin should not be surprising in the presence of all of the above factors that could contribute to poorer outcomes.

However, in a Cochrane review of randomized controlled trials (RCTs), the effectiveness and safety of Ceftriaxone or Cefotaxime were compared with conventional treatment with Penicillin or Ampicillin plus Chloramphenicol in patients with communityacquired acute bacterial meningitis. No clinically important difference between Ceftriaxone or Cefotaxime and conventional antibiotics was identified. Pelkonen T. et al in the study among the Sub-Saharan Africa children also showed the evidence that supported the finding of a Cochrane review above in that, treatment with ceftriaxone instead of with the primary regimen of Penicillin plus Chloramphenicol, did not improve the prognosis.

On the other hand, the report from KOSOVO showed that, the risk for developing neurologic complications and mortality was very high in patients treated with the initial antimicrobial therapy using Ceftriaxone alone or with Chloramphenicol than those initially treated with Penicillin G alone or with Chloramphenicol. Similarly, Theodoridou et al. also reported that, third-generation cephalosporin was related to an increased risk of hydrocephalus and ventriculitis, and the use of Penicillin was found to have a protective effect against neurologic sequelae (48). The strength of the last study in showing higher rates of neurotoxicity associated with cephalosporin compared to penicillin is that, it used multinomial logistic regression to identify the association among the different $\mathrm{AB}$ regimens with specific types of neurologic complications. But the current study used binomial logistic regression in that the association with neurotoxicity was not clearly known [48].

In the current study, another factor related to $A B$ s found to have a statistically significant association with poor outcomes was number of missed ABs dose. Patients who missed one or more doses of their prescribed $A B s$ experienced more pronounced poor outcomes than those never missed their $\mathrm{ABs}$ dose. As it was mentioned above under young infants section, this could highlight the need of intervention by narrowing the gap between the health care team and patients' family or caregivers by working together and searching for all possible options for the benefit of the patients.

\section{Strengths and limitations of the} study

Our study has some strength. Firstly, the design was prospective observational hospital based study in which most relevant variables were collected. Secondly, it was the first study in the country to study the outcomes of BM associated with $A B s$ use. However, our study was not free of limitation and needs precaution in interpreting. To mention some: the study period was short due to time limitation which couldn't allow collecting enough sample size, which resulted in wider ranges of confidence intervals. In addition, it would have been better if the patients were followed for some time after discharge to get the full impact of the disease so that complete outcomes could be measured. Because of that sever neurologic sequelae could not be detected within this short period; instead the data were limited to short-term acute complications.

The other important factor common to most resource limited settings was the lack of availability of some important laboratory facilities in which most of the cases of the current study were not confirmed by laboratory evidence, instead of the clinical diagnosis which may be less accurate than the laboratory assisted one.

Lastly, lack of studies regarding sensitivity of causative agents to the existing antimicrobials in our setups could impact treatment as well as comparisons of the regimens in this study. 


\section{Conclusion}

In the current study the selection of empiric therapy, change of empiric regimen as well as the timing of antimicrobials for treatment of childhood BM was almost consistent with recommendations for resource limited settings except that the timing of empiric antibiotics change was much longer. At discharge nearly one-fourth of the patients treated for BM experienced poor outcomes.

Finally, some independent predictors of poor outcomes were identified. In young infants, change of empiric antibiotics during the course of treatment was found to independently predict the incidence of poor outcomes. Whereas, in older infants and children, severe clinical presentations characterized by irritability and seizure prior hospital admission and drug related factors including initial treatment with a combination of Ceftriaxone and Gentamycin instead of first line Crystalline penicillin plus Chloramphenicol and missing one or more doses of the prescribed antibiotics during the course of treatment were found to independently increase incidence of poor outcomes.

\section{Recommendations}

The hospital has to create awareness of the health care team to give due attention for patients presented with sever clinical features like seizure and irritability as these were the alarming signs of poor outcomes. The hospital and concerned bodies should also follow and strengthen effective communication between the health care team and patients (family/care givers) since their non-compliance (missing their prescribed $A B s$ doses) were found to implicate poor prognosis. As this can be solved by improving the interaction between the patient (family/care givers) and the health care team to create common understanding for the benefit of the patient, thereby searching for and facilitating all the possible options for the patient (family/care givers) on how to get the cost effective alternatives. In addition, the study could highlight the need for revising management protocols regarding timing of the change of empiric $\mathrm{ABs}$ for children with $\mathrm{BM}$ in this ward.

The current study could also highlight that the need for further study with large sample size and longer study period and other studies that focuses on antimicrobial sensitivity in the setup.

\section{Ethics approval and consent to participate}

Ethical clearance was obtained from the institutional review board of Jimma University, college of health sciences. Patient consent was obtained from care givers or family in a written form by 2 local languages (Afan Oromo and Amharic) prior data collection.

\section{Availability of data and material}

The datasets generated and/or analyzed during the current study are not publicly available, but are available from the corresponding author on reasonable request.

\section{Competing interests}

The authors declared that they have no competing interests.

\section{Funding}

No specific funding was received for this work.

\section{Authors' contributions}

HA designed the study, carried out an analysis and interpreted the patient data, and drafted the manuscript. $\mathrm{SH}$ participated in the design of the study, supervised, coordinated and helped to draft the manuscript. LC participated in the design of the study, supervised, coordinated and helped to draft the manuscript. All authors read and approved the final manuscript.

\section{Acknowledgements}

First of all we would like to acknowledge JU and JUSH for permitting us to conduct the study and JUSH pediatric ward staffs for their support during data collection. Furthermore, our sincere appreciation also goes to our study participants and their family/care givers who voluntarily and genuinely gave their time as well as information. 


\section{REFERENCES}

Kliegman RM, Behrman RE, Jenson HB. Nelson text book of pediatrics (chapter-602, HTML). 18th ed. (2008).

Boyles TH, Bamford C, Bateman K, et al. Guidelines for the management of acute meningitis in children and adults in South Africa. South Afr. J. Epidemiol. Infect. 28(1), 5-15 (2013).

Ahmed A. Etiology of Bacterial Meningitis in Ethiopia, 2007-2011: A Retrospective Study(A thesis). 2012.

Kim KS. Acute bacterial meningitis in infants and children. Lancet Infect. Dis. 10(1), 32-42 (2010).

Antoniuk SA, Hamdar F, Ducci RD, Kira ATF, Cat MNL, Cruz CR. Childhood acute bacterial meningitis : Risk factors for Acute Neurological Complications and Neurological Sequelae. J. Pediatr. (Rio J). 87(6), 535-540 (2011).

Ansari I, Pokhrel Y. Culture Proven Bacterial Meningitis in Children : Agents, Clinical Profile and Outcome. Kathmandu Univ. Med. J. 33(1), 36-40 (2011).

Juganariu G, Miftode E, Teodor D. Clinical features and course of becterial meningitis in children. Med. Chir. Soc. Med. Nat. Iaş̧i. 116(3), 722-726 (2012).

Tebruegge M, Curtis N. Epidemiology, Etiology, Pathogenesis, and Diagnosis of Recurrent Bacterial Meningitis. Clin. Microbiol. Rev. 21(3), 519-537 (2008).

Namani S, Koci BM, Milenković Z, et al. Early neurologic complications and long-term sequelae of childhood bacterial meningitis in a limited-resource country (Kosovo). Child's Nerv. Syst. 29(2), 275280 (2013).

World Health Organization. Antimicrobial and support therapy for bacterial meningitis in children; Report, Geneva, Switzerland. Food, Drug, cosmetic law quarterly, WHO/ CHD/98.6. 1997.

Molyneux EM, Walsh AL, Forsyth $\mathrm{H}$, Tembo M, Mwenechanya J, Kayira $\mathrm{K}$, et al. Dexamethasone treatment in childhood bacterial meningitis in Malawi : a randomised controlled trial. Lancet. 360 , 211-218 (2002).
Peltola H. Burden of Meningitis and Other Severe Bacterial Infections of Children in Africa: Implications for Prevention. Clin. Infect. Dis. 32(1), 64-75 (2001).

Fortnum HM, Davis AC. Epidemiology of bacterial meningitis. Arch. Dis. Child. 68(1), 763-767 (1993).

Muluneh D, Shimelis D, Benti D. Analysis of admissions to the pediatric emergency ward of Tikur Anbessa Hospital in Addis Ababa , Ethiopia. Ethiop. J. Heal. Dev. 21(1), 48-55 (2007).

Sudharshan Raj. C PRMN. Pattern and antibiogram of bacterial meningitis in children at a tertiary care hospital. J. Sci. Innov. Res. 2(6), 1012-1016 (2013).

Ethiopia - FMHACA. Standard Treatment Guidelines for General Hospitals, 3rd edition. 388-390 (2014).

Tunkel AR, Hartman BJ, Kaplan SL, et al. Practice Guidelines for the Management of Bacterial Meningitis. IDSA guidelines. Clin. Infect. Dis. 39(1), 1267-1284 (2004).

Furyk JS, Swann O, Molyneux E. Systematic review: Neonatal meningitis in the developing world. Trop. Med. Int. Heal. 16(6), 672-679 (2011).

World Health Organization. Pocket Book of Hospital care for children. 55 \& 167 (2013).

Ethiopia M of $\mathrm{H}$. Pocket Book of Pediatric Hospital Care: 1 st edition. 65 \& 187 (2010).

Cha S, Mccracken GH. Bacterial Meningitis in Children. Pediatr. Clin. N. Am. 52(1), 795-810 (2005).

Deribew A, Tessema F, Girma B. Determinants of under-five mortality in Gilgel Gibe Field. Ethiop. J. Heal Dev. 21(2), 1-8 (2007).

Tegene B, Gebreselassie S, Fikrie N. Bacterial Meningitis: A Five-Year Retrospective Study Among Patients Who Had Attended at University of Gondar Teaching Hospital , Northwest Ethiopia. Biomed. Res. Ther. 2(5), 270-278 (2015).

Amsalu S1 AA. Meningitis in Children Beyond The Neonatal Period in Gondar
University Hospital. Ethiop. Med. J. 43(3), 175-180 (2005).

Jimma University (2015) Jimma University Specialized Hospital; available at: www.ju.edu.et.

Kurt N, Aydogan A, Ecevit C, Anlar Y, Gulumser O, Tanir G, et al. A Prospective Study of Etiology of Childhood Acute Bacterial, Turky. Emerg. Infect. Dis. 14(7), 1089-1096 (2008).

Hughes JB. Clinical Review: Evidence behind the WHO Guidelines: What are the Useful Clinical Features of Bacterial Meningitis Found in Infants and Children? J. Trop. Pediatr. 54(2), 83-86 (2008).

Saux N Le, Society CP. Guidelines for the management of suspected and confirmed bacterial meningitis in Canadian children older than one month of age. Paediatr. Child Heal. 19(3), 141146 (2014).

Shin SH, Kim KS. Treatment of Bacterial Meningitis: An Update. Expert Opin. Pharmacother. 13(15), 2189-2206 (2012).

Yogev R, Guzman-cottrill J. Bacterial Meningitis in Children Critical Review of Current Concepts. Adis. Data Inf. BV. 65(8), 1097-1112 (2005).

Pelkonen T, Roine I, Monteiro L, et al. Risk factors for death and severe neurological sequelae in childhood bacterial meningitis in Sub-Saharan Africa. Clin. Infect. Dis. 48(8), 1107-1110 (2009).

Namani S, Milenkovi Z, Koci B. A prospective study of risk factors for neurological complications in childhood bacterial meningitis. J. Pediatr. (Rio J). 89(3), 256-262 (2013).

Namani S, Koci R, Dedushi K. The outcome of bacterial meningitis in children is related to the initial antimicrobial therapy. Turk. J. Pediatr. 52(4), 354-359 (2010).

Degli Atti MC, Esposito S, Parola L, et al. In-hospital management of children with bacterial meningitis in Italy. Ital. J. Pediatr. 40(87) (2014).

Kiwanuka JP, Mwanga J. Childhood 
bacterial meningitis in Mbarara Hospital, Uganda: Antimicrobial Susceptibility and Outcome of Treatment. Afr. Heal. Sci. 1(1), 9-11 (2001).

Society CP, Committee (CPS); Infectious Diseases and Immunization. Therapy of suspected bacterial meningitis in Canadian children six weeks of age and older. Paediatr. Child Health. 13(4), 309 (2008)

Ramakrishnan M, Ulland AJ, Steinhardt LC, et al. Sequelae due to bacterial meningitis among African children : a systematic literature review. BMC Med. 7(47), 1741-7015 (2009).

38 Salih MA, Khaleefa OH, Bushara $\mathrm{M}$, et al. Long term sequelae of childhood acute bacterial meningitis in a developing country. A study from the Sudan. Scand. J. Infect. Dis. 23(2), $175-$ 182 (1991).

Rabbani MA, Khan AA, Ali SS, et al. Spectrum of Complications and Mortality of Bacterial Meningitis : an Experience from a Developing Country.
J. Pak. Med. Assoc. 53(12), 53-58 (2003).

Vasilopoulou VA, Karanika M, Theodoridou K, Katsioulis AT, Theodoridou MN, Hadjichristodoulou CS. Prognostic factors related to sequelae in childhood bacterial meningitis. BMC Infect. Dis. 11(1), 214-219 (2011).

Farag HFM, Abdel-Fattah MM, Youssri AM. Epidemiological, clinical and prognostic profile of acute bacterial meningitis among children in Alexandria, Egypt. Indian J. Med. Microbiol. 23(2), 95-101 (2005).

Laman M, Manning L, Greenhill AR, et al. Predictors of Acute Bacterial Meningitis in Children from a Malaria-Endemic Area of Papua New Guinea. Am. J. Trop. Med. Hyg. 86(2), 240-245 (2012).

Dzupova O, Rozsypal H, Prochazka $\mathrm{B}$, Benes J. Acute bacterial meningitis in adults: predictors of outcome. Scand. J. Infect. Dis. 41(5), 348-354 (2009).

Türel O, Yıldırım C, Yılmaz Y, et al. Clinical characteristics and prognostic factors in childhood bacterial meningitis: a multicenter study. Balkan Med. J. 30(1), 80-84 (2013).

Lin M, Chi H, Chiu N, Huang F. Factors for poor prognosis of neonatal bacterial meningitis in a medical center in Northern Taiwan. J. Microbiol. Immunol. Infect. 45(6), 442-247 (2012).

Gil Klinger, Choy-Nyok Chin, Joseph Beyene MP. Predicting the Outcome of Neonatal Bacterial Meningitis. Am. Acad. Pediatr. 106(3), 477-482 (2000).

Prasad K, Kumar A, Singhal T GP. Third generation cephalosporins versus conventional antibiotics for treating acute bacterial meningitis. Cochrane Database Syst. Rev. 2(1), 4 (2007).

Theodoridou K, Vasilopoulou VA, Katsiaflaka A, et al. Association of treatment for bacterial meningitis with the development of sequelae. Int. Soc. Infect. Dis. 17(9), e707-713 (2013)..

\section{This special issue on Current Trends in Clinical Research was edited by Dr. Mohamed Elsayed.}

